\title{
Uncoordinated Firing Rate Changes of Striatal Fast-Spiking Interneurons during Behavioral Task Performance
}

\author{
Joshua D. Berke \\ Department of Psychology, and Neuroscience Program, University of Michigan, Ann Arbor, Michigan 48109
}

\begin{abstract}
Basal ganglia circuits make key contributions to decision making. Distributed, synchronous feedforward inhibition of striatal medium spiny neurons by fast-spiking GABAergic interneurons (FSIs) has been argued to be important for the suppression of unwanted actions, and a deficit in FSIs has been found in human patients with Tourette syndrome. However, no studies have yet examined how striatal FSIs change their activity during behavioral tasks. Here I describe 36 presumed striatal FSIs recorded in rats during well practiced performance of a radial maze win-stay task. Although most FSIs showed robust task-related activity, the temporal patterns of firing rate change were highly idiosyncratic. In contrast to other classes of striatal neurons, FSIs showed little or no coordinated population response to major task events such as instruction cues or rewards. Even when multiple FSIs were recorded simultaneously from the same local region of striatum, firing rate changes were dissimilar, and no clear evidence for synchronous firing was found using cross-correlograms (18 FSI pairs examined). These results suggest that FSIs play a more complex role in the information processing achieved by striatal microcircuits than supposed by current theoretical models.
\end{abstract}

Key words: striatum; fast-spiking; interneuron; win-stay; feedforward; inhibition

\section{Introduction}

Cortical-basal ganglia circuits have a central role in the organization of behavior, including action selection and reinforcement learning (Schultz, 1998; Berke, 2008). Action selection involves enabling one option while suppressing others, to avoid the simultaneous selection of incompatible alternatives (Redgrave et al., 1999). Just how the basal ganglia contribute to this function is poorly understood. The most numerous cells in the basal ganglia are striatal medium spiny neurons (MSNs), GABAergic neurons whose axons form the striatal output and also innervate nearby MSNs. Influential earlier theories of striatal function (for review, see Wilson, 2000) argued that striatal MSNs form a competitive network via mutual lateral inhibition, whereby neurons participating in "winning" actions would naturally suppress representations of alternative actions. However, subsequent studies showed that axon collaterals of MSNs primarily target distal dendrites, and the resulting IPSPs are weak and nonreciprocal when recorded at the soma of target MSNs (Jaeger et al., 1994; Tunstall et al., 2002; Koos et al., 2004).

Such findings have highlighted the potential role of striatal interneurons in action selection. Parvalbumin-positive $(\mathrm{PV}+)$ fast-spiking interneurons (FSIs) are highly sensitive to cortical

Received May 14, 2008; revised Aug. 6, 2008; accepted Sept. 2, 2008.

This work was supported by grants from the National Institute on Drug Abuse, the National Institute of Mental Health, and the Tourette Syndrome Association. I thank Howard Eichenbaum for his generous support and guidance, Jason Breck for assistance with data analysis, Jennifer Skurski and Lotus McDougal for technical assistance, and Peter Magill, Paul Apicella, Greg Gage, and Michal Zochowski for comments on this manuscript. I also thank Avrama Blackwell and Jeanette Hellgren Kotaleski for sharing unpublished modeling results.

Correspondence should be addressed to Joshua D. Berke, Department of Psychology, and Neuroscience Program, University of Michigan, 530 Church Street, Ann Arbor, Ml 48109. E-mail: jdberke@umich.edu.

D0I:10.1523/JNEUROSCI.2192-08.2008

Copyright $\odot 2008$ Society for Neuroscience $\quad 0270-6474 / 08 / 2810075-06 \$ 15.00 / 0$ inputs, and are thought to integrate information from wide cortical areas (Parthasarathy and Graybiel, 1997). They are coupled together by gap junctions on their dendrites (Kita et al., 1990), suggesting a coordinated role in intrastriatal information processing. Each FSI provides perisomatic GABAergic inputs to $>100$ nearby MSNs, and even single FSI spikes can significantly delay MSN spikes (Koós and Tepper, 1999). These properties suggest that FSIs are activated synchronously by their inputs and simultaneously inhibit a large number of their target MSNs.

Coordinated, widespread feedforward inhibition might assist with action selection in several ways. First, it could establish a distributed field of inhibition, through which only coherent cortical activity can break through to activate MSNs (Parthasarathy and Graybiel, 1997). This would help "keep the basal ganglia gate shut until competitive [cortical] dynamics ... have a chance to select a unique ... goal" (Brown et al., 2004). Second, blanket suppression of MSNs by FSIs may serve to reset the striatal network when switching between action representations (Plenz, 2003). Third, FSIs may sculpt narrow time windows for synaptic integration by MSNs (Pouille and Scanziani, 2001), because only MSNs that receive strong coincident input would reach firing threshold before being suppressed via feedforward inhibition. A role for FSIs in action selection is indirectly supported by behavioral evidence. A deficit in striatal PV + cells has been observed in a rodent model of paroxysmal dystonia [abnormal cocontractions of opposing muscles (Gernert et al., 2000)] and in humans with Tourette syndrome (Kalanithi et al., 2005).

One class of extracellularly recorded striatal neurons shows multiple traits characteristic of FSIs, including very brief spike waveforms, the ability to fire at high frequencies, and a graded distribution (Berke et al., 2004). Recently it was confirmed that extracellularly recorded rat striatal neurons with the narrowest 
waveforms are PV+ (Mallet et al., 2005). I therefore examined whether, in behaving rats, FSIs show coordinated population activity consistent with widespread feedforward inhibition to MSNs.

\section{Materials and Methods}

Behavioral task. Rats were trained to perform a win-stay task under computer control in a wooden plus maze, painted matte black, elevated $71 \mathrm{~cm}$ from the floor, and surrounded by dark curtains. The maze consisted of a central octagon $(25.5 \mathrm{~cm}$ diameter $)$, four arms $(46 \times 9.5 \mathrm{~cm})$, and four goal boxes at the end of the arms $(30 \times 15 \mathrm{~cm})$. Each component had 3 -cm-high walls. A CCD camera mounted on the ceiling tracked the position of infrared light-emitting diodes (LEDs) on the rats' headstage at $30 \mathrm{~Hz}$. A pair of cue lights (green LEDs on stalks, located on each arm $12 \mathrm{~cm}$ from the central octagon), indicated the rewarded arm on each trial; normally off, they began flashing ( $125 \mathrm{~ms}$ on, $75 \mathrm{~ms}$ off) as the rat approached the central octagon (triggered at $32.2 \mathrm{~cm}$ from the maze center). Cue lights were carefully arranged to be visible as soon as they started flashing, regardless of which other arm the rat was on. The goal boxes contained liquid dispensers that automatically rewarded correct performance with droplets (100-150 $\mu \mathrm{l}$ ) of slightly sweetened water (the water was dispensed as the rat entered the correct goal box). The task was continuous in that the goal box for each trial immediately became the start box for the next trial, and there were no barriers present to limit the rat's movement. The cued arm was selected pseudorandomly on each trial, with the constraint that if the rat had made the same egocentric (e.g., left turn) choice on the previous two trials, then the rat was not cued to make the same movement a third time in a row. Rats were allowed to run freely until they showed a prolonged pause without initiating another trial (typically, rats ran $\sim 100$ trials over $\sim 40$ min sessions).

Data acquisition and analysis. Electrophysiological procedures and recording locations have been published in detail previously (Berke et al., 2004); the great majority of the cells described here were also part of that earlier dataset. Extracellular spikes were amplified and filtered at $600-$ $6000 \mathrm{~Hz}$ or $300-6000 \mathrm{~Hz}$ by custom-built amplifiers, and digitized at $30.33 \mathrm{kHz}$. To avoid counting neurons more than once, spikes from a given electrode were only measured from a single behavioral session, unless the electrode had been moved at least $80 \mu \mathrm{m}$ between sessions. Criteria for distinguishing between cell types were similar to before (Berke et al., 2004). Cells with valley width (at half maximum) $>0.35 \mathrm{~ms}$ and mean firing rate $<10 \mathrm{~Hz}$ were presumed MSNs [none of these cells had the very long duration waveforms and broad initial gap in their autocorrelograms characteristic of monkey "tonically active neurons" (TANs)]. Cells with valley width $<0.24 \mathrm{~ms}$ and peak width $<0.11 \mathrm{~ms}$ were presumed fast-spiking interneurons for the reasons previously described (Berke et al., 2004; Mallet et al., 2005), provided that they were also near-continuously active [fraction of interspike interval (ISIs) longer than $1 \mathrm{~s}<2 \%$ ]. Of the remainder, cells with $0.20<$ valley width $<$ $0.29 \mathrm{~ms}$ and $0.11<$ peak width $<0.15 \mathrm{~ms}$ were termed $\mathrm{O}$ cells; these parameters described a rare cell class with a distinctive waveform shape (see Fig. 2A). Nine adult male Long-Evans rats contributed FSIs and $\mathrm{O}$ cells to the data presented here; seven of these rats were implanted with tetrodes (using $12.5 \mu \mathrm{m}$ nichrome wire) and two with bundles of stereotrodes (using $25 \mu \mathrm{m}$ nichrome wire). Thirty of thirty-six FSIs and four of five "O cells" were recorded with tetrodes. Analyses were performed in MATLAB or Neuroexplorer. Spatial firing maps were constructed by binning position values into a $128 \times 128$ pixel grid and averaging the firing rates across all visits to each pixel, followed by spatial smoothing by convolution with a Gaussian kernel $(5 \times 5$ pixels; $\sigma=2$ pixels). Pixels with less than three visits are shown in black. Bin sizes were $50 \mathrm{~ms}$ for perievent time histograms and $500 \mu \mathrm{s}$ for cross-correlograms, without smoothing. Significance criterion for cross-correlogram peaks/ troughs was set at three successive bins outside the $99.5 \%$ confidence limits of the mean.

\section{Results}

\section{Classification of striatal neurons}

Rat striatal and hippocampal neurons were recorded during well practiced performance (76-99\% correct) of a radial maze task, in

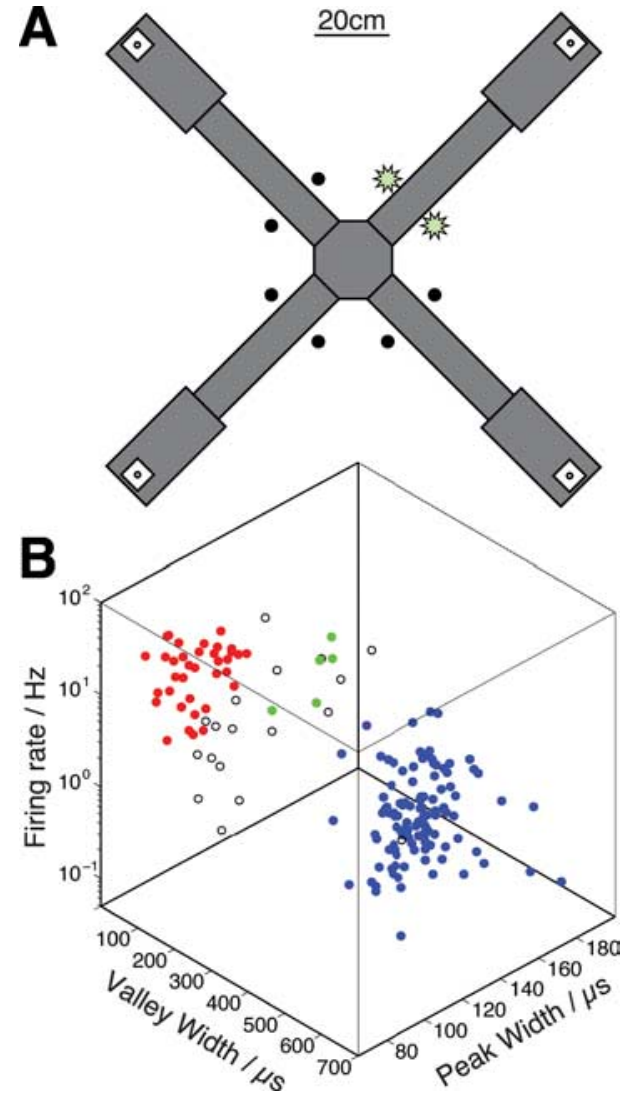

Figure 1. Win-stay task and striatal cell classification. $A$, Plus-maze arrangement (to scale). Thirsty rats ran continuously between water ports at the arm ends. As the rat approached the central zone on each trial, one pair of lights began flashing to indicate which arm choice would be rewarded. $\boldsymbol{B}$, Striatal neurons fall into distinct classes based on waveform characteristics [see also Fig. 2 and Berke et al. (2004)]. Most striatal neurons had wide waveforms (blue dots) and were not tonically active; these are presumed MSNs. One set of tonically active cells had very brief waveforms (red dots) and are presumed fast-spiking GABAergic interneurons. Another, rarely encountered set of tonically active neurons (green dots; " 0 cells") with distinctive waveforms may represent another interneuron class (see Results). Black circles indicate unclassified neurons. Mean peak width, valley width, and session-wide firing rate for each cell class were as follows: MSNs, $141 \mu \mathrm{s}, 474 \mu \mathrm{s}, 1.39 \mathrm{~Hz}$; FSIs, $87 \mu \mathrm{s}, 144 \mu \mathrm{s}, 24.7 \mathrm{~Hz} ; 0$ cells, $128 \mu \mathrm{s}, 252 \mu \mathrm{s}$, $15.2 \mathrm{~Hz}$. Six additional unclassified units were excluded from this plot because unusually complex waveform shapes prevented unequivocal assignment of "peak" and "valley" labels to waveform components.

which thirsty subjects ran between water ports with light cues indicating which maze arm choice would be rewarded (Fig. 1A). This is a continuous version of the "win-stay" task that has previously been shown to be dependent on intact striatal function (McDonald and White, 1993). Of 175 distinct striatal neurons that were sufficiently active and stable to analyze further, 109 were judged to be MSNs, and 36 were judged to be FSIs (Fig. $1 B$ ). The task-related firing patterns of striatal and hippocampal projection cells will be reported separately. In addition, a rare $(n=5)$ class of active neurons was identified with intermediate valley widths and a characteristic waveform shape (Fig. 2A). The distinct properties of these other cells ("O cells") are described below.

As previously reported (Berke et al., 2004), FSIs showed a dramatic state-dependent shift in firing pattern. In awake, behaving animals, FSIs tonically fired individual spikes, but during slow-wave sleep (SWS) they switched to an irregular burst mode that resulted in a narrow, early $(<10 \mathrm{~ms})$ peak in their interspike interval distribution (Fig. 2A). This early peak was very promi- 


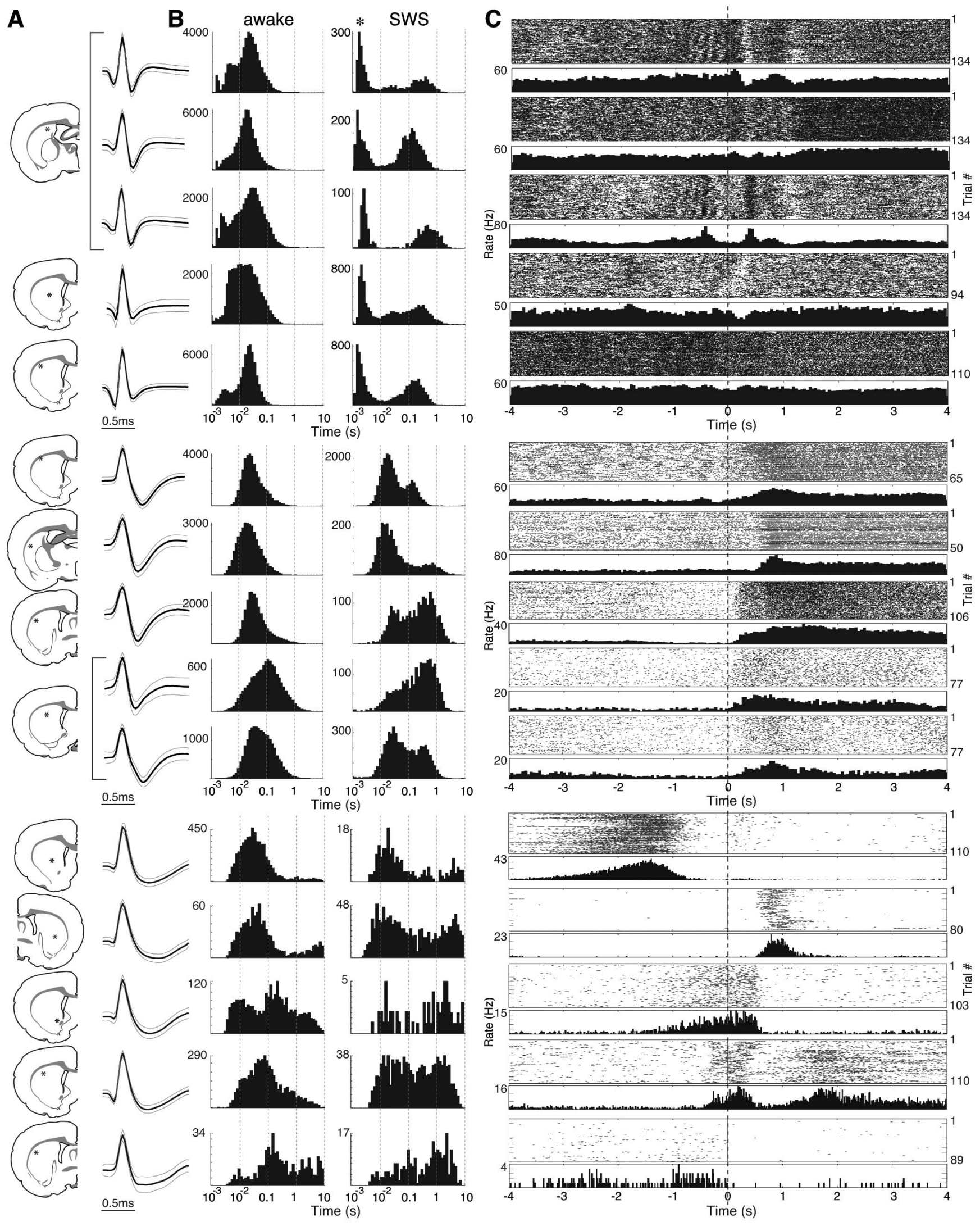

Figure 2. Distinct activity patterns and behavioral correlates of presumed striatal neuron subpopulations. $\boldsymbol{A}$, Five examples each of FSIs (top), 0 cells (middle), and MSNs (bottom), showing recording locations in striatum and spike waveforms (left; mean \pm SD). $\boldsymbol{B}$, Interspike interval (ISI) histograms during task performance (awake) and SWS, for the same units as shown in $\boldsymbol{A}$. $y$-Axis is counts per bin (starting at zero). FSIs showed an early peak (marked with asterisk) in the ISI histogram during sleep. C, Corresponding raster plots (top panels) and perievent time histograms (bottom panels) aligned on arrivals at the baited reward port. Numbers at left of histograms indicate firing rate (in hertz; scales begin at zero); numbers at right of rasters indicate trial number. Examples illustrate the wide range of distinct firing rate changes observed for FSIs and MSNs, whereas all 0 cells had similar firing rate time courses. The top three FSIs were recorded simultaneously from the same tetrode in dorsal striatum, demonstrating that nearby FSIs can have very different patterns of behavior-linked firing rate change. 
nent in 16 of 17 FSIs examined during SWS (94\%; from six different rats), but was never observed for $\mathrm{O}$ cells or MSNs (Fig. 2A).

When the relationships between cell classes and local field potential oscillations were examined, FSIs and $\mathrm{O}$ cells were found to fire at distinct phases of highvoltage spindles and sleep slow waves (supplemental Fig. 1, available at www. jneurosci.org as supplemental material). These findings further demonstrate that classifying striatal units on the basis of waveform shape can effectively demarcate distinct populations of striatal cells.

\section{Diverse FSI firing rate changes during task performance}

The behavioral correlates of putative striatal interneurons were first examined using perievent time histograms (PETHs) aligned on two key moments during each trial: the onset of the instruction cue and the arrival of the rat at the water reward. All O cells showed a similar pattern of firing rate change: relatively lower activity as rats ran along maze arms and then a marked and prolonged increase after reward receipt (Fig. 2C). This suggests that, in this well learned task, $\mathrm{O}$ cells are providing a relatively uniform signal as part of striatal information processing. In contrast, FSIs showed highly idiosyncratic firing rate changes (Fig. 2C). Although approximately three-quarters of the FSIs showed clear increases or decreases in firing in reward-aligned PETHs, these changes did not share a clear common direction, moment within trial, or characteristic duration.

To test whether the diversity of FSI firing rate changes was masking a common or predominant epoch of modulation, population-level PETHs were constructed by normalizing each cell to its mean firing rate and averaging the resulting individual PETHs (Fig. 3A). MSNs, FSIs, and O cells showed very different population activity time courses. The within-class similarity of $\mathrm{O}$ cell activity generated a population time course with a sharp increase after reward receipt, and reward-enhanced activity was also seen for MSNs. No such change occurred for FSIs, for which the population activity remained within $15 \%$ of the mean throughout both instruction-cue-aligned and reward-receiptaligned PETHs (Fig. 3A). When population activity was examined as a function of space instead of event-related time, a similar result was seen (Fig. 3B): whereas the activity of the MSN population was enhanced around the center of the maze and the reward ports, and the $\mathrm{O}$ cells were markedly more active at the reward ports, the FSI population showed a uniform coverage of the radial maze. This provides further evidence that there were no particular moments during the performance of this task that were associated with a coordinated change in FSI firing rate.

Evidence against a coordinated FSI feedforward signal within local striatal domains

The results above argue against a globally coordinated signal provided by striatal FSIs in this task. However, if broadly tuned feedforward signals operated within local functional domains of the striatum, one might not expect FSIs from different striatal subregions to have similar firing patterns. Particular attention was therefore paid to those cases in which multiple FSIs were simultaneously recorded from the same probe, and thus were very close together within striatum. Twelve such FSI pairs were identified (from four tetrode cases with two FSIs each, two tetrode cases with three FSIs each, and two stereotrode cases with two FSIs each). For 11/12 pairs, FSI firing rate modulation was dramatically dissimilar for the two FSIs (for example, see the top three cells of Fig. $2 \mathrm{~B}$ ). The remaining case was a pair in which neither cell showed rapid modulation of firing rate (both cells showed a slow increase during reward consumption; data not shown).

The possible synchronization of FSIs on finer time scales was examined by constructing cross-correlograms between pairs of FSIs in the time range $[-20 \mathrm{~ms}+20 \mathrm{~ms}]$. No significant peaks or troughs were seen for any of the 12 FSI pairs recorded simultaneously from the same probe (supplemental Fig. 2, available at www.jneurosci.org as supplemental material). The only exceptions were two sessions in which cross-correlograms showed a centered narrow peak in the $<3 \mathrm{~ms}$ range. These early peaks were considered an unreliable indicator of synchrony for cells recorded from the same probe, because the inability to separate overlapping spikes produces such peaks through a "shadowing effect" (Bar-Gad et al., 2003) even for uncorrelated neurons. This peak was never seen for FSI pairs recorded from separate probes (0/6 pairs) (supplemental Fig. 2, available at www.jneurosci.org as supplemental material). Thus, no compelling evidence was seen for short time-scale synchronization between striatal FSIs during behavioral task performance, with the caveat that technical limitations degrade detection of possible synchrony at $<3 \mathrm{~ms}$ for neurons recorded from the same probe. 


\section{Discussion}

How the dynamic properties of forebrain microcircuits contribute to mental/behavioral functions is a major current area of neuroscientific investigation. In the striatum, anatomical studies and electrophysiological work in slices and anesthetized animals have demonstrated that divergent feedforward inhibition via FSIs is a potentially powerful component of intrastriatal information processing (Pennartz and Kitai, 1991). On this basis, it has been proposed that the coordinated activity of FSIs plays an important role in choices, by broad suppression of MSNs involved in unselected actions or goals. The present work set out to test whether FSIs in fact show coordinated changes in firing rate in awake, behaving animals. Surprisingly, presumed FSIs showed highly independent firing rate changes, even within very local regions of striatum. Although data are shown here only from a single, well learned task, at no point during task performance or reward consumption was there a consistent change in FSI firing rate that would indicate a coordinated response. The rich and diverse activity time courses of most FSIs suggest that these cells are active participants in the details of striatal input:output transformations, rather than providing a global signal involved in (for example) broad suppression of unselected actions, or network reset.

This diversity of FSI firing patterns stands in marked contrast to the more uniform activity of other important components of striatal information processing. In particular, the signals provided by both the mesostriatal dopamine input and intrastriatal cholinergic signaling are generally considered to be far more scalar in nature (Schultz, 1998; Morris et al., 2004). Although some monkey "TANs" (presumed cholinergic interneurons) show selectivity in their responses [for example, to contralaterally vs ipsilaterally presented stimuli (Ravel et al., 2006)], the onset time, direction, and duration of their pause-rebound firing rate changes are quite consistent between cells. Another likely subtype of striatal interneurons, termed O cells here, also showed a consistent pattern of firing rate changes (increased activity after reward receipt). This result is intriguing but must be viewed with caution, because the number of these neurons recorded is small and their identity currently unknown. Because they have briefer waveforms than MSNs (but not so brief as FSIs), I speculate that they represent one of the other known subpopulations of striatal GABAergic interneurons, such as those that colocalize somatostatin/nitric oxide synthase/NPY, or calretinin. However, insights into the functional role of this population within striatal microcircuitry will have to wait until their chemical phenotype is established and their activity examined in a wider range of behavioral situations. It is worth noting that cells with the longduration waveform and regular spike pattern characteristic of monkey TANs (presumed cholinergic interneurons) were almost never encountered during these experiments. Conversely, presumed GABAergic interneurons have not been reported in monkey studies. Although PV + cells are a larger proportion of interneurons in rats than in primates (Wu and Parent, 2000), such cross-species differences do not seem sufficient to account for this; differences in the electrode types used and neuronal morphology (Yelnik et al., 1993) may be contributing factors.

In sensory regions of cortex, FSIs mediate feedforward inhibition from thalamus, enforcing the temporal precision of principal cell responses to external stimuli (Wehr and Zador, 2003; Cruikshank et al., 2007). Within a single somatosensory barrel, cortical FSIs each receive powerful inputs from overlapping sets of thalamic neurons, resulting in a synchronized FSI population with high sensitivity but low specificity [i.e., broad tuning (Swad- low, 2003)]. Striatal FSIs possess similar anatomical and physiological features, including gap junction coupling, convergent inputs, and divergent outputs, that suggest they too should act as a temporally coordinated population. The apparent lack of synchronization between striatal FSIs was therefore quite unexpected. However, recent modeling studies of the striatal FSI network have found that gap junctions do not, in fact, dramatically enhance synchrony in response to synaptic inputs (J. Hjorth, K. Blackwell, and J. Hellgen Kotaleski, personal communication). Rather, their dominant impact is to lower overall firing rates through current shunting, except in the case in which FSIs receive highly synchronous inputs. Consistent with this, we have previously observed that striatal FSIs do fire synchronously during high-voltage spindles, an $\sim 8 \mathrm{~Hz}$ idling rhythm that broadly engages cortex, basal ganglia, and thalamus (Berke et al., 2004; Dejean et al., 2007).

The dissimilar behavioral response patterns of striatal FSIs might reflect the fractured afferent topography of this structure, with different FSIs located in distinct functional domains (Wilson, 2000). However, the dendrites and axons of FSIs do not respect known intrastriatal compartment boundaries such as those between patches (striosomes) and matrix (Cowan et al., 1990; Bennett and Bolam, 1994). Further, dissimilar firing rate changes were seen even for FSIs that very likely had overlapping dendritic and axonal fields. Although the effective "range" of microelectrodes for detecting cells in striatum is not known, in a hippocampal study using the same type of tetrodes as used here, effective unit separation from noise was obtained only when the probe was $<50 \mu \mathrm{m}$ from the cell soma (Henze et al., 2000). Therefore, in the present study, FSIs simultaneously recorded from the same probe were likely $<100 \mu \mathrm{m}$ apart, and are likely to be influencing overlapping sets of MSNs. FSIs have been estimated to make synapses on $\sim 25 \%$ of MSNs within $250 \mu \mathrm{m}$ of their cell body, and each MSN receives synapses from 4-27 FSIs (Koós and Tepper, 1999). The present results therefore suggest that individual MSNs receive inputs from FSIs with very different behavioral correlates.

If each MSN receives converging inputs from a substantial number of distinct FSIs with uncorrelated firing rate changes, the overall rate of GABAergic inputs to a given MSN would remain nearly constant during task performance. Although this arrangement could certainly provide broadly tuned inhibition, preventing weak cortical activity from evoking spikes in MSNs, it begs the question of why there should be extrinsic inputs to FSIs at all. Unlike some other classes of tonically active basal ganglia neurons, striatal FSIs are silent if deprived of their inputs (Plenz and Kitai, 1998). Compared with MSNs, which receive sparse inputs from many different cells (Kincaid et al., 1998), FSIs seem to be driven by a far smaller number of distinct corticostriatal neurons, from multiple cortical areas onto individual FSIs (Ramanathan et al., 2002). Clearly it would be helpful to obtain more information about the subpopulation of cortical neurons that project to FSIs.

In addition, FSIs receive a specific (GABAergic) projection from globus pallidus (Bevan et al., 1998). The function of this "return" projection is not known, although it has been suggested to act as one of a set of signals providing dynamic gain control (Gurney and Overton, 2004). Because neurons in globus pallidus also show distinctive and uncorrelated firing rate changes (BarGad et al., 2003), this projection may well contribute to the observed striatal FSI firing patterns, although it is not possible with these methods to determine the relative contributions of glutamatergic and GABAergic inputs. If the globus pallidus input exerts a strong influence over the firing pattern of striatal FSIs, then it might make more sense to conceptualize these cells as part of a 
feedback pathway within the basal ganglia rather than, or in addition to, providing feedforward inhibition from cortex. Although feedforward inhibition via striatal FSIs is clearly present after artificial stimulation of cortex (Mallet et al., 2005), the overall notion of feedforward inhibition is perhaps less compelling when the striatum is viewed as one component of cortex-basal ganglia-thalamus-cortex loops whose recurrent nature is critical to their normal operation (Leblois et al., 2006).

Finally, it is important to consider the various time scales over which striatal FSI activity might be coordinated. Fast-spiking GABAergic interneurons contribute to the orchestration of projection neuron spike timing in many brain regions (Buzsáki, 2006), and striatal FSIs are in a commanding position to control the fine spike timing of MSNs (Koos et al., 2004). FSIs can show entrainment to fast oscillations in the local field potential (Berke, 2005) and may be vital for the recruitment of local MSN populations into ensembles. It is therefore possible that the most significant aspects of FSI activity, including synchronization, operate on the millisecond time scale. Although no convincing evidence was found for synchronization between pairs of striatal FSIs, session-wide cross-correlograms may not have detected transient forms of synchrony that occur around specific key behavioral or neural events (Paz et al., 2006). The examination of FSI:FSI and FSI:MSN relationships during behavioral tasks in which decisions can be analyzed with high temporal precision will be an important area for future studies.

\section{References}

Bar-Gad I, Heimer G, Ritov Y, Bergman H (2003) Functional correlations between neighboring neurons in the primate globus pallidus are weak or nonexistent. J Neurosci 23:4012-4016.

Bennett BD, Bolam JP (1994) Localisation of parvalbuminimmunoreactive structures in primate caudate-putamen. J Comp Neurol 347:340-356

Berke JD (2005) Participation of striatal neurons in large-scale oscillatory networks. In: The basal ganglia VIII (Bolam JP, Ingham CA, Magill PJ, eds), pp 25-35. New York: Springer.

Berke JD (2008) Procedural learning: striatum. In: Encyclopedia of neuroscience (Squire LR, Albright T, Bloom FE, Gage FH, Spitzer N, ed). Oxford: Elsevier.

Berke JD, Okatan M, Skurski J, Eichenbaum HB (2004) Oscillatory entrainment of striatal neurons in freely moving rats. Neuron 43:883-896.

Bevan MD, Booth PA, Eaton SA, Bolam JP (1998) Selective innervation of neostriatal interneurons by a subclass of neuron in the globus pallidus of the rat. J Neurosci 18:9438-9452.

Brown JW, Bullock D, Grossberg S (2004) How laminar frontal cortex and basal ganglia circuits interact to control planned and reactive saccades. Neural Netw 17:471-510.

Buzsáki G (2006) Rhythms of the brain. New York: Oxford UP.

Cowan RL, Wilson CJ, Emson PC, Heizmann CW (1990) Parvalbumincontaining GABAergic interneurons in the rat neostriatum. J Comp Neurol 302:197-205.

Cruikshank SJ, Lewis TJ, Connors BW (2007) Synaptic basis for intense thalamocortical activation of feedforward inhibitory cells in neocortex. Nat Neurosci 10:462-468.

Dejean C, Gross CE, Bioulac B, Boraud T (2007) Synchronous high-voltage spindles in the cortex-basal ganglia network of awake and unrestrained rats. Eur J Neurosci 25:772-784.

Gernert M, Hamann M, Bennay M, Löscher W, Richter A (2000) Deficit of striatal parvalbumin-reactive GABAergic interneurons and decreased basal ganglia output in a genetic rodent model of idiopathic paroxysmal dystonia. J Neurosci 20:7052-7058.

Gurney KN, Overton PG (2004) A model of short and long range selective processing in neostriatum. Neurocomputing 58-60:555-562.

Henze DA, Borhegyi Z, Csicsvari J, Mamiya A, Harris KD, Buzsáki G (2000) Intracellular features predicted by extracellular recordings in the hippocampus in vivo. J Neurophysiol 84:390-400.

Jaeger D, Kita H, Wilson CJ (1994) Surround inhibition among projection neurons is weak or nonexistent in the rat neostriatum. J Neurophysiol 72:2555-2558.

Kalanithi PS, Zheng W, Kataoka Y, DiFiglia M, Grantz H, Saper CB, Schwartz ML, Leckman JF, Vaccarino FM (2005) Altered parvalbumin-positive neuron distribution in basal ganglia of individuals with Tourette syndrome. Proc Natl Acad Sci U S A 102:13307-13312.

Kincaid AE, Zheng T, Wilson CJ (1998) Connectivity and convergence of single corticostriatal axons. J Neurosci 18:4722-4731.

Kita H, Kosaka T, Heizmann CW (1990) Parvalbumin-immunoreactive neurons in the rat neostriatum: a light and electron microscopic study. Brain Res 536:1-15.

Koós T, Tepper JM (1999) Inhibitory control of neostriatal projection neurons by GABAergic interneurons. Nat Neurosci 2:467-472.

Koos T, Tepper JM, Wilson CJ (2004) Comparison of IPSCs evoked by spiny and fast-spiking neurons in the neostriatum. J Neurosci 24:7916-7922.

Leblois A, Boraud T, Meissner W, Bergman H, Hansel D (2006) Competition between feedback loops underlies normal and pathological dynamics in the basal ganglia. J Neurosci 26:3567-3583.

Mallet N, Le Moine C, Charpier S, Gonon F (2005) Feedforward inhibition of projection neurons by fast-spiking GABA interneurons in the rat striatum in vivo. J Neurosci 25:3857-3869.

McDonald RJ, White NM (1993) A triple dissociation of memory systems: hippocampus, amygdala, and dorsal striatum. Behav Neurosci 107:3-22.

Morris G, Arkadir D, Nevet A, Vaadia E, Bergman H (2004) Coincident but distinct messages of midbrain dopamine and striatal tonically active neurons. Neuron 43:133-143.

Parthasarathy HB, Graybiel AM (1997) Cortically driven immediate-early gene expression reflects modular influence of sensorimotor cortex on identified striatal neurons in the squirrel monkey. J Neurosci 17:2477-2491.

Paz R, Pelletier JG, Bauer EP, Paré D (2006) Emotional enhancement of memory via amygdala-driven facilitation of rhinal interactions. Nat Neurosci 9:1321-1329.

Pennartz CM, Kitai ST (1991) Hippocampal inputs to identified neurons in an in vitro slice preparation of the rat nucleus accumbens: evidence for feed-forward inhibition. J Neurosci 11:2838-2847.

Plenz D (2003) When inhibition goes incognito: feedback interaction between spiny projection neurons in striatal function. Trends Neurosci 26:436-443.

Plenz D, Kitai ST (1998) Up and down states in striatal medium spiny neurons simultaneously recorded with spontaneous activity in fast-spiking interneurons studied in cortex-striatum-substantia nigra organotypic cultures. J Neurosci 18:266-283.

Pouille F, Scanziani M (2001) Enforcement of temporal fidelity in pyramidal cells by somatic feed-forward inhibition. Science 293:1159-1163.

Ramanathan S, Hanley JJ, Deniau JM, Bolam JP (2002) Synaptic convergence of motor and somatosensory cortical afferents onto GABAergic interneurons in the rat striatum. J Neurosci 22:8158-8169.

Ravel S, Sardo P, Legallet E, Apicella P (2006) Influence of spatial information on responses of tonically active neurons in the monkey striatum. J Neurophysiol 95:2975-2986.

Redgrave P, Prescott TJ, Gurney K (1999) The basal ganglia: a vertebrate solution to the selection problem? Neuroscience 89:1009-1023.

Schultz W (1998) Predictive reward signal of dopamine neurons. J Neurophysiol 80:1-27.

Swadlow HA (2003) Fast-spike interneurons and feedforward inhibition in awake sensory neocortex. Cereb Cortex 13:25-32.

Tunstall MJ, Oorschot DE, Kean A, Wickens JR (2002) Inhibitory interactions between spiny projection neurons in the rat striatum. J Neurophysiol 88:1263-1269.

Wehr M, Zador AM (2003) Balanced inhibition underlies tuning and sharpens spike timing in auditory cortex. Nature 426:442-446.

Wilson CJ (2000) Striatal circuitry: categorically selective, or selectively categorical? In: Brain dynamics and the striatal complex (Miller R, Wickens JR, eds). Amsterdam: Harwood.

Wu Y, Parent A (2000) Striatal interneurons expressing calretinin, parvalbumin or NADPH-diaphorase: a comparative study in the rat, monkey and human. Brain Res 863:182-191.

Yelnik J, Percheron G, François C, Garnier A (1993) Cholinergic neurons of the rat and primate striatum are morphologically different. Prog Brain Res 99:25-34. 